\title{
Finite-time Control for a Class of Networked Control Systems with Short Time-varying Delays and Sampling Jitter
}

\author{
Chang-Chun Hua ${ }^{1} \quad$ Shao-Chong $\mathrm{Yu}^{1} \quad$ Xin-Ping Guan ${ }^{1,2}$ \\ ${ }^{1}$ Department of Electrical Engineering, University of Yanshan, Qinhuangdao 066004, China \\ ${ }^{2}$ Department of Automation, Shanghai Jiaotong University, Shanghai 200240, China
}

\begin{abstract}
This paper is concerned with the finite-time control problem for a class of networked control systems (NCSs) with short time-varying delays and sampling jitter. Considering a state feedback controller, the closed-loop NCS is described as a discrete-time linear system model, and the uncertain parts reflect the effect of the the network-induced delays and short sampling jitter of the system dynamics. Then a robust approach is proposed to solve the finite-time stability and stabilization problems for the considered NCS. An illustrative example is provided to demonstrate the effectiveness of the proposed theoretical results.
\end{abstract}

Keywords: Networked control system (NCS), time-varying delay, sampling jitter, finite-time stability, state feedback.

\section{Introduction}

In a networked control system, one of the main problems is network-induced delay, and it is usually regarded as the major cause of deterioration of system performance and potential system instability. Since the network and the scheduling policies in the networks are varying, the networkinduced delay is typically varying. Compared with the constant delay, the time-varying delay is more difficult to deal with. For the past decade, various approaches have been presented in the existing literature to deal with the modeling, analysis, and synthesis problems for the networked control systems (NCSs) with delays. To deal with short time-varying delays, one effective approach is the uncertain system approach ${ }^{[1-6]}$. The main idea of this approach is that by decomposing the time-varying network-induced delay into the nominal part and the uncertain part, the NCSs can be described as a class of discrete norm bounded uncertain systems. Therefore, the stabilizing state feedback controller can be designed based on the uncertain system approach. The stabilization problem is considered for the class of wireless networked control systems (WNCS). ${ }^{[7]}$

In the sampling process, the clock circuit of the sensor in the NCS may be affected by some external influence such as high-frequency electric signals and temperature. As a consequence, the sampling period may be not the constant

\footnotetext{
Regular paper

Manuscript received January 3, 2013; accepted November 4, 2013

This paper was supported by National Natural Science Foundation of China (Nos.61290322, 61273222, 61322303 and 61473248), Doctoral Fund of Ministry of Education of China (No. 20121333110008), Hebei Province Hundred Excellent Innovation Talents Support Program, and Hebei Province Applied Basis Research Project (No. 13961806D).

Recommended by Associate Editor Qing-Long Han

(C) Institute of Automation, Chinese Academy of Science and Springer-Verlag Berlin Heidelberg 2015
}

which we set, but a value changing in a range, i.e., there exists sampling jitter. However, if the sampling period jitter is large enough, the considering NCS may be unstable. Therefore, how to design the controller to ensure the stability and dynamic performance of NCSs in this situation is a problem worth considering. To solve this problem, it is necessary and great significant to establish the relation between the stability of the NCSs and the sampling jitter. By the sampled-data control system approach, we can establish the relation between the stability and the sampling period. The sampling jitter problem was discussed in sampled-data control systems, but the main idea was different and the time-varying delays were not considered ${ }^{[8,9]}$. Therefore, new methods need to be explored to model and analyze the NCSs with both short time-varying delays and sampling jitter. This motivates the present research.

Besides, most of the results in the literature are focused on the Lyapunov stability of the NCSs. Often asymptotic stability is enough for practical applications, but there are some cases where large values of the state are not acceptable, for instance in the presence of saturations. In these cases, we need to check that these unacceptable values are not attained by the state, for these purposes, finite-time stability (FTS) can be used. The concept of Lyapunov asymptotic stability is widely known to the control community. Conversely, a system is said to be finite-time stable if its state does not exceed some bounds during this timeinterval. The finite-time stability, or short-time stability was proposed during the $1960 \mathrm{~s}^{[10,11]}$. In recent years, the concept of finite-time stability has been revisited in the light of linear matrix inequality theory. Lots of valuable results have been obtained for this type of stability ${ }^{[12-17]}$. Some sufficient conditions for finite-time stability and stabilization of continuous systems or discrete-time systems were 
provided $^{[12-14]}$. Feng et al. ${ }^{[15-17]}$. extended the definition of finite-time stability to the systems with impulsive effects or singular systems with impulsive effect, respectively, and derived some sufficient conditions for finite-time stability and stabilization problems. The finite-time stability approach was extended into the area of the switched linear system, and some sufficient conditions were provided for finitetime stability and stabilization problems ${ }^{[18,19]}$. In this paper, we deal with the finite-time control problems of the NCSs with short time-varying delays and the sampling jitter. A new uncertain system model is proposed to describe the considered NCS with sampling jitter, and the uncertainties of the jitter and delays are expressed as the uncertainties of the system matrices. Similar modeling methods have been presented ${ }^{[3,14,15]}$. However, sampling jitter and external disturbance have not been considered ${ }^{[3,14,15]}$. Additionally, the definition of finite-time stability is recalled and extended to the discrete-time NCSs. Then, a sufficient condition for the existence of a state feedback controller which guarantees the finite-time boundedness of the NCS is given.

The paper is organized as follows. Section 2 is devoted to preliminaries and problem formulation. The main results for FTS analysis are given in Section 3. A numerical example is presented to illustrate the efficiency of the proposed method in Section 4. Finally, the conclusions are given in Section 5 .

\section{Preliminaries and problem formula- tion}

Consider an NCS model with the structure shown in Fig. 1, where the continuous-time plant is described by the linear time-invariant system model as

$$
\begin{aligned}
& \dot{x}(t)=A_{p} x(t)+B_{p} u(t)+G \omega(t) \\
& \dot{\omega}(t)=F \omega(t)
\end{aligned}
$$

where $x(t) \in \mathbf{R}^{n}$ and $u(t) \in \mathbf{R}^{m}$ are the system state and the control input, $\omega(t) \in \mathbf{R}^{q}$ is the exogenous input, $A_{p}, B_{p}, G, F$ are a set of known real constant matrices with appropriate dimensions. Throughout the paper, the following assumptions are needed for the considered NCS.

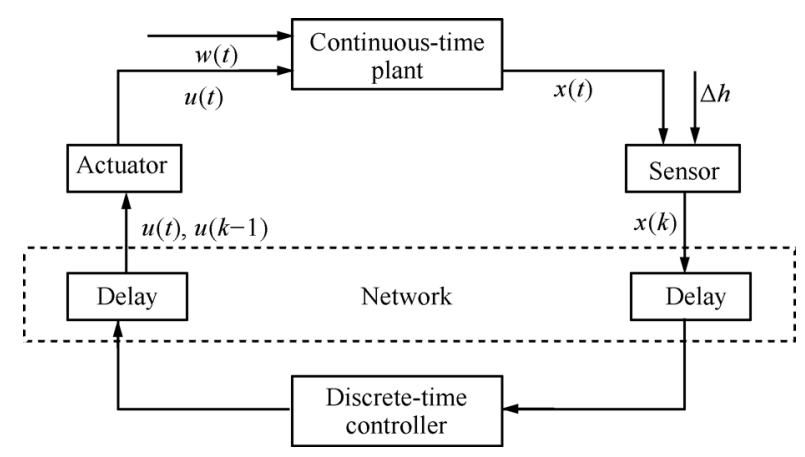

Fig. 1 The structure of NCS with short time varying delays
Assumption 1. The sensor is time-driven and both the controller and the actuator are event-driven.

Assumption 2. The unknown time-varying networkinduced delay at time step $k$ is denoted by $\tau_{k}, \tau_{k}=$ $\tau_{s c}(k)+\tau_{c a}(k)$ is smaller than a sampling period and is upper bounded by $\tau_{k} \leq \bar{\tau} \leq h, \tau_{s c}(k)$ and $\tau_{c a}(k)$ are the sensor-to-controller delay and the controller to actuator delay, respectively. There is no packet dropout in the networks.

Assumption 3. Consider that the setting sampling period is $h_{0}$, and the real time-varying sampling period is denoted by $h=h_{0}+\Delta h$, where $-h_{0}<-\Delta \bar{h} \leq \Delta h \leq \Delta \bar{h}<h_{0}$ and $\Delta \bar{h}$ is the upper bound of the sampling jitter.

Remark 1. Since the sampling jitter is caused by the external interference acting on the sensor, the size of the jitter is unknown. But based on the actual situation of the sensor, it can be of sufficiently small size, and it is definite that the jitter may vary the sampling period in both larger and smaller directions. Therefore, we assume that $-\Delta \bar{h} \leq$ $\Delta h \leq \Delta \bar{h}$, and $\Delta \bar{h}$ is far less than $h_{0}$.

Discretizing the system given by (1) with the period of $h$ and taking the network-induced delay $\tau_{k}$ into account, we obtain the discrete model

$$
\begin{aligned}
& x(k+1)=A x(k)+B_{0}\left(\tau_{k}\right) u(k)+B_{1}\left(\tau_{k}\right) u(k-1)+G \omega \\
& \omega(k+1)=F \omega(k)
\end{aligned}
$$

where

$$
A=\mathrm{e}^{A_{p} h}
$$

and

$$
\begin{aligned}
& B_{0}\left(\tau_{k}\right)=\int_{0}^{h-\tau_{k}} \mathrm{e}^{A_{p} s} \mathrm{~d} s B_{p} \\
& B_{1}\left(\tau_{k}\right)=\int_{h-\tau_{k}}^{h} \mathrm{e}^{A_{p} s} \mathrm{~d} s B_{p} .
\end{aligned}
$$

Denote $A_{0}=\mathrm{e}^{A_{p} h_{0}}$. Then, by (2), we have

$$
\begin{aligned}
A= & \mathrm{e}^{A_{p} h}=\mathrm{e}^{A_{p} h_{0}}+\left(\mathrm{e}^{A_{p} h}-\mathrm{e}^{A_{p} h_{0}}\right)= \\
& \mathrm{e}^{A_{p} h_{0}}+\mathrm{e}^{A_{p} h_{0}}\left(\mathrm{e}^{A_{p} \Delta h}-I\right)
\end{aligned}
$$

when $\Delta h$ is sufficiently small. By Taylor formula ,we have that $\mathrm{e}^{A_{p} \Delta h}=A_{p} \Delta h+I$, so that we obtain

$$
\begin{aligned}
A= & \mathrm{e}^{A_{p} h_{0}}+\mathrm{e}^{A_{p} h_{0}}\left(A_{p} \Delta h+I-I\right)= \\
& \mathrm{e}^{A_{p} h_{0}}+\mathrm{e}^{A_{p} h_{0}} A_{p} \Delta h= \\
& A_{0}+A_{0} \Delta h A_{p}= \\
& A_{0}+A_{0} \Theta_{0}(\Delta h) A_{p}= \\
& A_{0}+\Delta A
\end{aligned}
$$

where

$$
\Theta_{0}(\Delta h)=\Delta h, \quad \Delta A=A_{0} \Theta_{0}(\Delta h) A_{p} .
$$


Let $B_{0}=\int_{0}^{h_{0}} \mathrm{e}^{A_{p} s} \mathrm{~d} s B_{p}$, we can obtain that

$$
\begin{aligned}
B_{0}\left(\tau_{k}\right)= & \int_{0}^{h-\tau_{k}} \mathrm{e}^{A_{p} s} \mathrm{~d} s B_{p}= \\
& \int_{0}^{h_{0}+\Delta h-\tau_{k}} \mathrm{e}^{A_{p} s} \mathrm{~d} s B_{p}= \\
& \int_{0}^{h_{0}} \mathrm{e}^{A_{p} s} \mathrm{~d} s B_{p}+\int_{h_{0}}^{h_{0}+\Delta h-\tau_{k}} \mathrm{e}^{A_{p} s} \mathrm{~d} s B_{p}= \\
& B_{0}+A_{0} \int_{0}^{\Delta h-\tau_{k}} \mathrm{e}^{A_{p} s} \mathrm{~d} s B_{p}= \\
& B_{0}+A_{0} \Theta_{1}\left(\Delta h, \tau_{k}\right) B_{p}= \\
& B_{0}+\Delta B
\end{aligned}
$$

where

$$
\begin{aligned}
& \Theta_{1}\left(\Delta h, \tau_{k}\right)=\int_{0}^{\Delta h-\tau_{k}} \mathrm{e}^{A_{p} s} \mathrm{~d} s \\
& \Delta B=A_{0} \Theta_{1}\left(\Delta h, \tau_{k}\right) B_{p} .
\end{aligned}
$$

Let $B_{2}=\int_{0}^{h} \mathrm{e}^{A_{p} s} \mathrm{~d} s B_{p}$, we have that

$$
\begin{aligned}
B_{2}= & \int_{0}^{h} \mathrm{e}^{A_{p} s} \mathrm{~d} s B_{p}= \\
& \int_{0}^{h_{0}+\Delta h} \mathrm{e}^{A_{p} s} \mathrm{~d} s B_{p}= \\
& \int_{0}^{h_{0}} \mathrm{e}^{A_{p} s} \mathrm{~d} s B_{p}+\int_{h_{0}}^{h_{0}+\Delta h} \mathrm{e}^{A_{p} s} \mathrm{~d} s B_{p}= \\
& \int_{0}^{h_{0}} \mathrm{e}^{A_{p} s} \mathrm{~d} s B_{p}+\mathrm{e}^{A_{p} h_{0}} \int_{0}^{\Delta h} \mathrm{e}^{A_{p} s} \mathrm{~d} s B_{p}= \\
& B_{0}+A_{0} \Theta_{2}\left(\Delta h, \tau_{k}\right) B_{p}=B_{0}+\Delta \tilde{B}
\end{aligned}
$$

where

$$
\begin{aligned}
& \Theta_{2}\left(\Delta h, \tau_{k}\right)=\int_{0}^{\Delta h} \mathrm{e}^{A_{p} s} \mathrm{~d} s \\
& \Delta \tilde{B}=A_{0} \Theta_{2}\left(\Delta h, \tau_{k}\right) .
\end{aligned}
$$

Since $B_{2}=B_{0}\left(\tau_{k}\right)+B_{1}\left(\tau_{k}\right)$, we have $B Z_{1}\left(\tau_{k}\right)=B_{2}-$ $B_{0}\left(\tau_{k}\right)=B_{0}+\Delta \tilde{B}-B_{0}-\Delta B=\Delta \tilde{B}-\Delta B$. Then, with the state feedback controller $u(k)=K x(k)$, the closed-loop NCS can be described as

$$
\begin{aligned}
& x(k+1)=A_{c} x(k)+B_{c} x(k-1)+G \omega(k) \\
& \omega(k+1)=F \omega(k)
\end{aligned}
$$

where

$$
\begin{aligned}
& A_{c}=A_{0}+B_{0} K+A_{0} \Theta_{0}(\Delta h) A_{p}+A_{0} \Theta_{1}\left(\Delta h, \tau_{k}\right) B_{p} K \\
& B_{c}=A_{0} \Theta_{2}\left(\Delta h, \tau_{k}\right) B_{p} K-A_{0} \Theta_{1}\left(\Delta h, \tau_{k}\right) B_{p} K .
\end{aligned}
$$

The definitions of finite-time stability and finite-time boundedness in NCSs are given below.

Definition $\mathbf{1}^{[\mathbf{1 4}]}$. When $\omega(k) \equiv 0$, the discrete-time linear NCS given in (3) is said to be finite-time stable with respect to $\left(\delta_{x}, \epsilon, R, N\right)$, where $R$ is a positive-definite matrix, $0<\delta_{x}<\epsilon$, and $N \in \mathbf{N}_{0}$, if

$$
x^{\mathrm{T}}(0) R x(0) \leq \delta_{x}^{2} \Rightarrow x^{\mathrm{T}}(k) R x(k)<\epsilon^{2}, \quad \forall k \in\{1, \cdots, N\} .
$$

Definition $2^{[14]}$. The discrete-time linear system given in (3) is said to be finite-time bounded with respect to $\left(\delta_{x}, \epsilon, R, N\right)$, where $R$ is a positive-definite matrix, $0 \leq \delta_{x}<$ $\epsilon, \delta_{\omega} \geq 0$, and $N \in \mathbf{N}_{0}$, if

$$
\begin{gathered}
x^{\mathrm{T}}(0) R x(0) \leq \delta_{x}^{2} \\
\omega^{\mathrm{T}}(0) \omega(0) \leq \delta_{\omega}^{2}
\end{gathered} \quad \Rightarrow x^{\mathrm{T}}(k) R x(k)<\epsilon^{2}, \forall k \in\{1, \cdots, N\} .
$$

Given system (3), our main aim is to find some sufficient conditions which guarantee that the system given by (3) is bounded over a finite-time interval. The general idea of finite-time stability concerns the roundedness of the state of a system over a finite time interval for given initial conditions. So we need to solve the following problem.

Problem 1. Designing a state feedback controller of the form $u(k)=K x(k)$ such that the closed-loop NCS (3) is finite-time bounded with respect to $\left(\delta_{x}, \epsilon, R, N\right)$.

Remark 2. In this proposed modeling method, the uncertainties of sampling jitter and the network-induced delay are transformed into the uncertainties of the system matrices.

\section{Finite-time stability analysis and controller design}

In this section, a finite-time stability criteria for the NCS (5) is given, as well as a sufficient condition for the existence of the state feedback stabilizing controller. To obtain the results, the following lemma will be needed.

Lemma 1. For the known constant matrices $Y=Y^{\mathrm{T}}, D$ and $E$ with appropriate dimensions, if

$$
Y+D F E+E^{\mathrm{T}} F^{\mathrm{T}} D^{\mathrm{T}}<0
$$

where $F$ satisfies that $F^{\mathrm{T}} F \leq \delta^{2} I$ and $\delta$ is a finite constant, then there exist constants $\varepsilon>0$ and $\mu>0$, such that

$$
Y+\varepsilon D D^{\mathrm{T}}+\mu E^{\mathrm{T}} E<0 .
$$

Theorem 1. System (3) is FTS with respect to $\left(\delta_{x}, \delta_{\omega}, \epsilon, R, N\right)$ if there exist positive-definite matrices $P_{1} \in$ $\mathbf{R}^{n \times n}, P_{2} \in \mathbf{R}^{n \times n}$ and $P_{3} \in \mathbf{R}^{n \times n}$, and scalars $\varepsilon>0, \mu>0$ and $\gamma \geq 1$, such that

$\left[\begin{array}{ccccc}\Pi_{1} & 0 & 0 & \left(A_{0}+B_{0} K\right)^{\mathrm{T}} & A_{p}^{\mathrm{T}}+K^{\mathrm{T}} B_{p}^{T} \\ * & \Pi_{2} & 0 & 0 & K^{\mathrm{T}} B_{p}^{\mathrm{T}} \\ * & * & \Pi_{3} & G^{\mathrm{T}} & 0 \\ * & * & * & -P_{1}^{-1}+\varepsilon A_{0} A_{0}^{\mathrm{T}} & 0 \\ * & * & * & * & -\mu I\end{array}\right]<0$

$$
\begin{aligned}
& \left(\lambda_{\max }\left(\tilde{P}_{1}\right) \delta_{x}^{2}+\lambda_{\max }\left(P_{3}\right) \delta_{\omega}^{2}\right) \gamma^{N}- \\
& \quad \lambda_{\min }\left(P_{3}\right) \lambda_{\min }(\tilde{F}) \delta_{\omega}^{2}<\epsilon^{2} \lambda_{\min }\left(\tilde{P}_{1}\right)
\end{aligned}
$$


where $\Pi_{1}=-\gamma P_{1}+P_{2}, \Pi_{2}=-\gamma P_{2}, \Pi_{3}=-\gamma P_{3}+$ $\mathrm{F}^{\mathrm{T}} P_{3} F, \tilde{P}_{1}=R^{-\frac{1}{2}} P_{1} R^{-\frac{1}{2}}$ and $\tilde{F}=\left(F^{\mathrm{T}}\right)^{N} F^{N}$.

Proof. Let us assume that $x^{\mathrm{T}}(0) R x(0) \leq \delta_{x}^{2}, x(-1)=0$ and $\omega^{\mathrm{T}}(0) \omega(0) \leq \delta_{\omega}^{2}$. Our aim is to prove that if condition (5) holds, then $x^{\mathrm{T}}(k) R x(k)<\epsilon^{2}$ for all $k=1, \cdots, N$.

Choose the Lyapunov-like function

$V(k)=x^{\mathrm{T}}(k) P_{1} x(k)+x^{\mathrm{T}}(k-1) P_{2} x(k-1)+\omega^{\mathrm{T}}(k) P_{3} \omega(k)$.

Denoting $\xi(k)=\left[x^{\mathrm{T}}(k) x^{\mathrm{T}}(k-1) \omega^{\mathrm{T}}(k)\right]^{\mathrm{T}}$, we have

$$
\begin{aligned}
& V(k+1)-\gamma V(k)= \\
& \quad x^{\mathrm{T}}(k+1) P_{1} x(k+1)+x^{\mathrm{T}}(k) P_{2} x(k)+ \\
& \omega^{\mathrm{T}}(k+1) P_{3} \omega(k+1)-x^{\mathrm{T}}(k) \gamma P_{1} x(k)- \\
& x^{\mathrm{T}}(k-1) \gamma P_{2} x(k-1)-\omega^{\mathrm{T}}(k) \gamma P_{3} \omega(k)= \\
& \xi^{\mathrm{T}}(k) \Psi \xi(k)
\end{aligned}
$$

where

$$
\begin{aligned}
\Psi= & {\left[\begin{array}{c}
A_{c}^{\mathrm{T}} \\
B_{c}^{\mathrm{T}} \\
G^{\mathrm{T}}
\end{array}\right]+P_{1}\left[\begin{array}{ccc}
A_{c} & B_{c} & G
\end{array}\right]+} \\
& {\left[\begin{array}{ccc}
-\gamma P_{1}+P_{2} & 0 & 0 \\
* & -\gamma P_{2} & 0 \\
* & * & -\gamma P_{3}+F^{\mathrm{T}} P_{3} F
\end{array}\right] . }
\end{aligned}
$$

$\Psi<0$ guarantees that $V(k+1)-\gamma V(k)<0$, which will be used for our proof. By Schur complement, $\Psi<0$ is equivalent to the following matrix inequality

$$
\left[\begin{array}{cccc}
-\gamma P_{1}+P_{2} & 0 & 0 & A_{c}^{\mathrm{T}} \\
* & -\gamma P_{2} & 0 & B_{c}^{\mathrm{T}} \\
* & * & -\gamma P_{3}+F^{\mathrm{T}} P_{3} F & G^{\mathrm{T}} \\
* & * & * & -P_{1}^{-1}
\end{array}\right]<0
$$

which can be written as

$$
\Psi_{0}+\bar{D} \Theta_{i}\left(\tau_{k}, \Delta h\right) \bar{E}+\bar{E}^{\mathrm{T}} \Theta_{i}^{\mathrm{T}}\left(\tau_{k}, \Delta h\right) \bar{D}^{\mathrm{T}}<0
$$

where

$$
\begin{aligned}
\Psi_{0} & =\left[\begin{array}{cccc}
-\gamma P_{1}+P_{2} & 0 & 0 & \left(A_{0}+B_{0} K\right)^{\mathrm{T}} \\
* & -\gamma P_{2} & 0 & 0 \\
* & * & -\gamma P_{3}+F^{\mathrm{T}} P_{3} F & G^{\mathrm{T}} \\
* & * & * & -P_{1}^{-1}
\end{array}\right] \\
\bar{D} & =\left[\begin{array}{cccc}
0 & 0 & 0 & A_{1}^{\mathrm{T}}
\end{array}\right]^{\mathrm{T}} \\
\bar{E} & =\left[\begin{array}{llll}
A_{p}+B_{p} K & B_{p} K & 0 & 0
\end{array}\right] .
\end{aligned}
$$

Since $\tau_{k}$ and $\Delta h$ are bounded, it can be known that $\Theta_{i}^{\mathrm{T}}\left(\tau_{k}, \Delta h\right) \Theta_{i}\left(\tau_{k}, \Delta h\right) \leq \delta^{2}$, where $\delta$ is a bounded constant. Therefore, by Lemma 1 , we have that

$$
\Psi_{0}+\varepsilon \bar{D} \bar{D}^{\mathrm{T}}+\mu \bar{E}^{\mathrm{T}} \bar{E}<0
$$

holds. It then follows from the Schur complement that (10) is equivalent to $(5 \mathrm{a})$.
Then, (5a) implies that

$$
V(k+1)<\gamma V(k) .
$$

Applying (11) iteratively and using the fact that $\gamma \geq 1$, we obtain

$$
\begin{aligned}
& V(k)<\gamma^{k} V(0)= \\
& \gamma^{k}\left(x^{\mathrm{T}}(0) P_{1} x(0)+x^{\mathrm{T}}(-1) P_{2} x(-1)+\omega^{\mathrm{T}}(0) P_{3} \omega(0)\right) \leq \\
& \gamma^{k}\left(\lambda_{\max }\left(\tilde{P}_{1}\right) x^{\mathrm{T}}(0) R x(0)+\lambda_{\max }\left(\tilde{P}_{2}\right) x^{\mathrm{T}}(-1) R x(-1)+\right. \\
& \left.\lambda_{\max }\left(\tilde{P}_{3}\right) \omega^{\mathrm{T}}(0) \omega(0)\right) \leq \\
& \gamma^{N}\left(\lambda_{\max }\left(\tilde{P}_{1}\right) x^{\mathrm{T}}(0) R x(0)+\lambda_{\max }\left(P_{3}\right) \omega^{\mathrm{T}}(0) \omega(0)\right) \leq \\
& \gamma^{N}\left(\lambda_{\max }\left(\tilde{P}_{1}\right) \delta_{x}^{2}+\lambda_{\max }\left(P_{3}\right) \delta_{\omega}^{2}\right), \\
& k=1, \cdots, N
\end{aligned}
$$

where $\tilde{P}_{1}=R^{-\frac{1}{2}} P_{1} R^{-\frac{1}{2}}, \tilde{P}_{2}=R^{-\frac{1}{2}} P_{2} R^{-\frac{1}{2}}$, $\lambda_{\max }\left(\tilde{P}_{1}\right), \lambda_{\max }\left(\tilde{P}_{2}\right), \lambda_{\max }\left(P_{3}\right)$ represent the maximum eigenvalues of matrices $\tilde{P}_{1}, \tilde{P}_{2}, P_{3}$, and

$$
\begin{aligned}
& V(k)=x^{\mathrm{T}}(k) P_{1} x(k)+x^{\mathrm{T}}(k-1) P_{2} x(k-1)+ \\
& \omega^{\mathrm{T}}(k) P_{3} \omega(k) \geq \\
& x^{\mathrm{T}}(k) P_{1} x(k)+\omega^{\mathrm{T}}(k) P_{3} \omega(k) \geq \\
& \lambda_{\min }\left(\tilde{P}_{1}\right) x^{\mathrm{T}}(k) R x(k)+\lambda_{\min }\left(P_{3}\right) \omega^{\mathrm{T}}(k) \omega(k) \geq \\
& \quad \lambda_{\min }\left(\tilde{P}_{1}\right) x^{\mathrm{T}}(k) R x(k)+\lambda_{\min }\left(P_{3}\right) \omega^{\mathrm{T}}(0)\left(F^{\mathrm{T}}\right)^{k} F^{k} \omega(0) .
\end{aligned}
$$

Denote $\tilde{F}=\left(F^{\mathrm{T}}\right)^{N} F^{N}$, and (13) can be described as

$$
\begin{aligned}
V(k) \geq & \lambda_{\min }\left(\tilde{P}_{1}\right) x^{\mathrm{T}}(k) R x(k)+\lambda_{\min }\left(P_{3}\right) \omega^{\mathrm{T}}(0) \tilde{F} \omega(0) \geq \\
& \lambda_{\min }\left(\tilde{P}_{1}\right) x^{\mathrm{T}}(k) R x(k)+ \\
& \lambda_{\min }\left(P_{3}\right) \lambda_{\min }(\tilde{F}) \omega^{\mathrm{T}}(0) \omega(0) \geq \\
& \lambda_{\min }\left(\tilde{P}_{1}\right) x^{\mathrm{T}}(k) R x(k)+\lambda_{\min }\left(P_{3}\right) \lambda_{\min }(\tilde{F}) \delta_{\omega}^{2}
\end{aligned}
$$

where $\lambda_{\min }(\cdot)$ represents the minimum eigenvalues of the corresponding matrix.

From (12) to (14), we obtain

$$
\begin{aligned}
& x^{\mathrm{T}}(k) R x(k)< \\
& \quad \frac{1}{\lambda_{\min }\left(\tilde{P}_{1}\right)}\left[\left(\lambda_{\max }\left(\tilde{P}_{1}\right) \delta_{x}^{2}+\lambda_{\max }\left(P_{3}\right) \delta_{\omega}^{2}\right) \gamma^{N}-\right. \\
& \left.\quad \lambda_{\min }\left(P_{3}\right) \lambda_{\min }(\tilde{F}) \delta_{\omega}^{2}\right] .
\end{aligned}
$$

From (15), it follows that (5b) implies that, for all $k=$ $1, \cdots, N, x^{\mathrm{T}}(k) R x(k)<\epsilon^{2}$. Then the close-loop NCS (3) is finite-time bounded with respect to $\left(\delta_{x}, \epsilon, R, N\right)$.

Remark 3 (Asymptotic stability from Theorem 1). If conditions (5) in Theorem 1 are satisfied by $\gamma=1$ and $\omega \equiv 0$, then the close-loop NCS (3) is finite-time stable with respect to $\left(\delta_{x}, \epsilon, R, N\right)$ for all $N \in \mathbf{N}_{0}$ and it is also asymptotically stable.

Remark 4. Compared with the proof in [14], we deal with $\omega^{\mathrm{T}}(k) P_{3} \omega(k)$ in (14) by a less conservative approach instead of simply discarding it. Therefore, the result we obtain in this paper has less conservatism. 
Theorem 2 (Controller design). System (3) is FTS with respect to $\left(\delta_{x}, \epsilon, R, N\right)$ if there exist positive-definite matrices $Q \in \mathbf{R}^{n \times n}, S \in \mathbf{R}^{n \times n}, T \in \mathbf{R}^{n \times n}$, matrix $U$, scalars $\varepsilon>0, \mu>0$ and $\gamma \geq 1$ such that

$$
\begin{gathered}
{\left[\begin{array}{ccccc}
\Gamma_{1} & 0 & 0 & Q A_{0}^{\mathrm{T}}+U^{\mathrm{T}} B_{0}^{\mathrm{T}} & Q A_{p}^{\mathrm{T}}+U^{\mathrm{T}} B_{p}^{\mathrm{T}} \\
* & \Gamma_{2} & 0 & 0 & U^{\mathrm{T}} B_{p}^{\mathrm{T}} \\
* & * & \Gamma_{3} & G^{\mathrm{T}} & 0 \\
* & * & * & -Q+\varepsilon A_{0} A_{0}^{\mathrm{T}} & 0 \\
* & * & * & * & -\mu I
\end{array}\right]<0} \\
\left(\lambda_{\max }(\tilde{Q}) \delta_{x}^{2}+\lambda_{\max }(T) \delta_{\omega}^{2}\right) \gamma^{N}-\lambda_{\min }(T) \lambda_{\min }(\tilde{F}) \delta_{\omega}^{2}< \\
\epsilon^{2} \lambda_{\min }(\tilde{Q})
\end{gathered}
$$

where $\Gamma_{1}=-\gamma Q+S, \Gamma_{2}=-\gamma S, \Gamma_{3}=-\gamma T+F^{\mathrm{T}} T F$, $\tilde{Q}=R^{-\frac{1}{2}} Q^{-1} R^{-\frac{1}{2}}$. Then NCS (3) is controlled by $u(k)=$ $K x(k)$, and the controller gain matrix is given by $K=$ $U Q^{-1}$.

Proof. Denoting $Q=P_{1}^{-1}, S=Q P_{2} Q, T=P_{3}$ and $U=K Q$, we obtain inequality (16a) by pre-multiplying and post-multiplying (5a) by $\operatorname{diag}\{Q, Q, I, I\}$ and (16b) by replacing $P_{1}, P_{2}, P_{3}$ by $Q, S$ and $T$.

Remark 5. Sun and $\mathrm{Xu}^{[20]}$ have considered the FTS problem of NCSs with constant time delays. This paper deals with the FTS problem of NCSs with time-varying delays. Compared with constant delay situation in [20], a time-varying delay situation in this paper is more coincident with the real network environment, and the processing of time-varying delays is an important part when we are analyzing NCSs. Besides, we have also considered the sampling jitter problem in this paper.

Remark 6. According to the procedures above, we have successfully extended the definition of FTS to NCS. Theorem 1 and Corollary 1 have been given to solve the stability and stabilization problems. Indeed, the key idea in our proof is to relax the condition of negative definiteness of $\Delta V: \Delta V$ does be not need to be negative definite but should just not greater than $(\gamma-1) V$. This is important for an NCS, because we can also give a controller to keep the system stable or bounded even that a negative definite $\Delta V$ cannot be found since the bad network environment or emergencies happen. To illustrate our results, a numerical example will be presented in the following section.

\section{Numerical example and simulations}

Example 1. Consider system (1), where

$$
\begin{aligned}
& A_{p}=\left[\begin{array}{cc}
2.9124 & 3.6276 \\
-3.6276 & -0.7152
\end{array}\right], \quad B_{p}=\left[\begin{array}{c}
-1.8138 \\
0.3576
\end{array}\right] \\
& G=\left[\begin{array}{ll}
1 & 0 \\
0 & 1
\end{array}\right], \quad F=\left[\begin{array}{cc}
0.8 & 0.6 \\
-0.6 & 0.8
\end{array}\right] .
\end{aligned}
$$

Choose the sampling period $h=0.5 \mathrm{~s}, 0<\tau_{k}<0.05 \mathrm{~s}, 0<$ $|\Delta h|<0.05 \mathrm{~s}, \delta_{x}=2, R=I$, and the initial system state $x(0)=\left[\begin{array}{ll}-1 & 0\end{array}\right]^{\mathrm{T}}, \omega(0)=\left[\begin{array}{ll}1 & 0\end{array}\right]^{\mathrm{T}}$. Then, the corre- sponding discrete system model (4) with short time-varying network-induced delay is given by

$$
\begin{aligned}
x(k+1)= & {\left[\begin{array}{cc}
1 & 2 \\
-2 & -1
\end{array}\right] x(k)+} \\
& \int_{0}^{0.5+\Delta h-\tau_{k}} \mathrm{e}^{A_{p} s} \mathrm{~d} s B_{p} u(k)+ \\
& \int_{0.5+\Delta h-\tau_{k}}^{0.5} \mathrm{e}^{A_{p} s} \mathrm{~d} s B_{p} u(k-1) .
\end{aligned}
$$

Case 1. When $\gamma=1, N=20, \omega=0, \delta_{x}=1$, we solved LMIs (16) and we found that the controller $K=$ $\left[\begin{array}{ll}0.71 & 1.11\end{array}\right]$ guarantees the desired closed-loop properties with $\epsilon=2.64$. The value of $\gamma$ (see Remark 3) implies that the state boundedness is guaranteed for all $N \in \mathbf{N}_{0}$ and that the closed-loop system is also asymptotically stable. The simulation results are shown in Figs. 2 and 3, which depict the state trajectories and the trajectories of $x(k) R x(k)$ when applying the designed controller.

Case 2. When $\gamma=1.3, N=4, \delta_{\omega}=1, \delta_{x}=1$, we solved LMIs (16) and found the controller $K=\left[\begin{array}{ll}1.44 & 1.60\end{array}\right]$ guarantees the desired closed-loop properties with $\epsilon=4.10$. From Figs. 4 and 5, it is straight to see that the states of the close-loop system are well bounded under the obtained controller gain during the specified time interval, which indicates the effectiveness of the theoretical results.

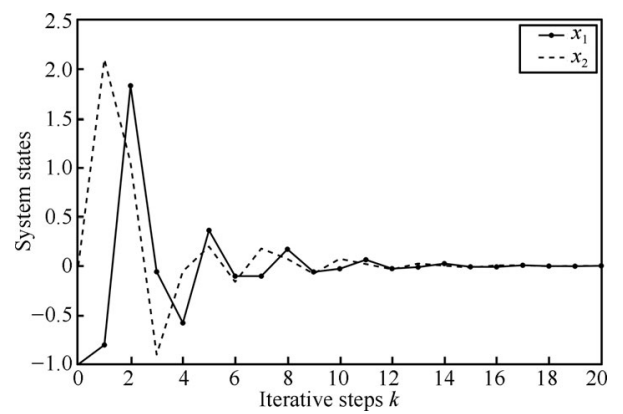

Fig. 2 The state trajectories at each iteration with $\gamma=1$

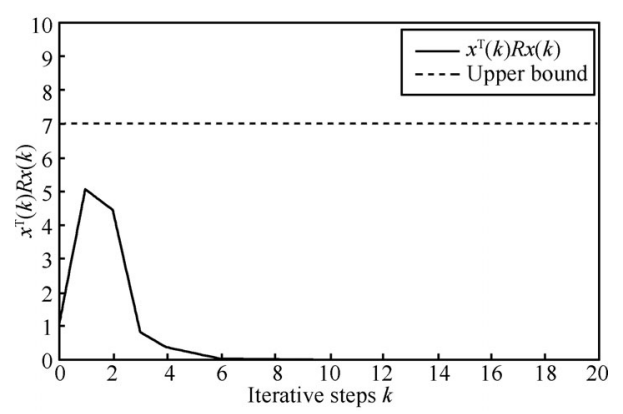

Fig. 3 The trajectory of $x^{\mathrm{T}}(k) R x(k)$ at each iteration with $\gamma=1$ 


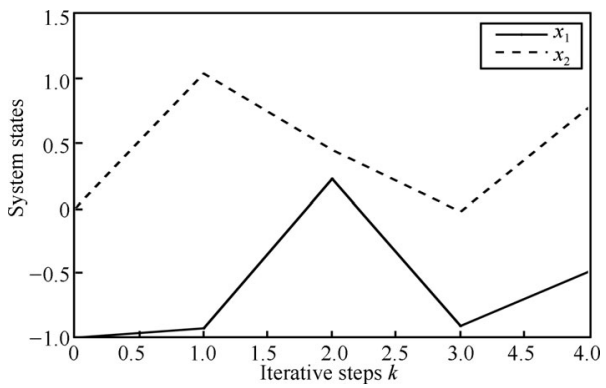

Fig. 4 The state trajectories at each iteration with $\gamma=1.3$

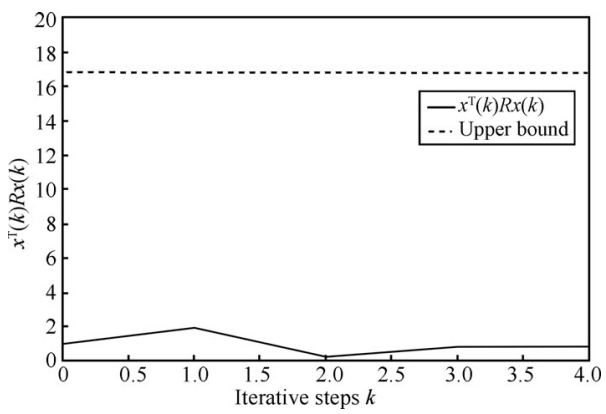

Fig. 5 The trajectory of $x^{\mathrm{T}}(k) R x(k)$ at each iteration with $\gamma=1.3$

\section{Conclusions}

This paper addresses the finite-time stabilization problem for a class of networked control systems with short time-varying delays and sampling jitter. A linear uncertain system model is proposed to describe the considered NCS. Starting from some conditions guaranteeing finite-time stability and finite-time boundedness, we have provided sufficient conditions for the state feedback problem by the robust control approach. The proposed design conditions are expressed in terms of linear matrix inequalities.

\section{References}

[1] L. Hetel, J. Daafouz, C. Lung. Analysis and control of LTI and switched systems in digital loops via an event-based modelling. International Journal of Control, vol. 81, no. 7, pp. 1125-1138, 2008 .

[2] P. Seiler, R. Sengupta. An $H_{\infty}$ approach to networked control. IEEE Transactions on Automatic Control, vol. 50, no. 3, pp. 356-364, 2005.

[3] M. B. G. Cloosterman, N. V. De Wouw, W. P. M. H. Heemels, H. Nijmeijer. Stability of networked control systems with uncertain time-varying delays. IEEE Transactions on Automatic Control, vol. 54, no. 7, pp. 1575-1580, 2009 .

[4] W. A. Zhang, L. Yu. A robust control approach to stabilization of networked control systems with time-varying delays. Automatica, vol. 45, no. 10, pp. 2440-2445, 2009.

[5] W. A. Zhang, L. Yu. A robust control approach to stabilization of networked control systems with short time-varying delays. Acta Automatica Sinica, vol. 36, no. 1, pp.87-91, 2010 .

[6] W. A. Zhang, L. Yu. BIBO stability and stabilization of networked control systems with short time-varying delays. International Journal of Robust and Nonlinear Control, vol. 21, no. 3, pp. 295-308, 2011.

[7] C. C. Hua, Y. Zheng, X. P. Guan. Modeling and control for wireless networked control system. International Journal of Automation and Computing, vol.8, no. 3, pp. 357-363, 2011.

[8] H. Fujioka. A discrete-time approach to stability analysis of systems with aperiodic sample-and-hold devices. IEEE Transactions on Automatic Control, vol. 54, no. 10, pp. 2440-2445, 2009.

[9] Y. Oishi, H. Fujioka. Stability and stabilization of aperiodic sampled-data control systems using robust linear matrix inequalities. Automatica, vol. 46, no. 8, pp. 1327-1333, 2010.

[10] P. Dorato. Short time stability in linear tine-varying systems. In Proceedings of the IRE International Convention Record Part 4, New York, USA, pp. 83-87, 1961.

[11] L. Weiss, E. Infante. Finite time stability under perturbing forces and on product spaces. IEEE Transactions on Automatic Control, vol. 12, no. 1, pp. 54-59, 1967.

[12] F. Amato, M. Ariola, P. Dorato. Finite-time control of linear systems subject to parametric uncertainties and disturbances. Automatica, vol. 37, no. 9, pp. 1459-1463, 2001.

[13] F. Amato, M. Ariola, C. Cosentino. Finite-time control of discrete-time linear systems: Analysis and design conditions. Automatica, vol. 46, no. 5, pp. 919-1924, 2010.

[14] F. Amato, M. Ariola. Finite-time control of discrete-time linear systems. IEEE Transactions on Automatic Control, vol. 50, no. 5, pp. 724-729, 2005.

[15] J. E. Feng, Z. Wu, J. B. Sun. Finite-time control of linear singular systems with parametric uncertainties and disturbances. Acta Automatica Sinica, vol. 31, no. 4, pp. 634-637, 2005. (in Chinese)

[16] L. Liu, J. Sun. Finite-time stabilization of linear systems via impulsive control. International Journal of Control, vol. 81, no. 6, pp. 905-909, 2008.

[17] S. W. Zhao, J. T. Sun, L. Liu. Finite-time stability of linear time-varying singular systems with impulsive effects. International Journal of Control, vol. 81, no. 11, pp. 1824-1829, 2008.

[18] H. B. Du, X. Z. Lin, S. H. Li. Finite-time stability and stabilization of switched linear systems. In Proceedings of the 48th IEEE Conference on Decision and Control and 28th Chinese Control Conference, IEEE, Shanghai, China, pp. 1938-1943, 2009. 
[19] H. Y. Song, L. Yu, W. A. Zhang. Finite-time $H_{\infty}$ control for a class of discrete-time switched time-delay systems with quantized feedback. Communications in Nonlinear Science and Numerical Simulation, vol.17, no. 12, pp. 4802-4814, 2012.

[20] Y. G. Sun, J. Xu. Finite-time boundedness and stabilization of networked control systems with time delay. Mathematical Problems in Engineering, vol. 2012, Article 705828, 2012.

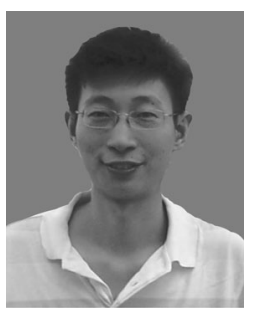

Chang-Chun Hua received the $\mathrm{Ph}$. D degree in electrical engineering from Yanshan University, China in 2005. He was a research fellow in National University of Singapore during 2006-2007. From 2007 to 2009, he worked in Carleton University, Canada, funded by Province of Ontario Ministry of Research and Innovation Program. From 2009 to 2011, he worked in University of Duisburg-Essen, Germany, funded by Alexander von Humboldt Foundation. Now, he is a full professor in Yanshan University, China. He is the author or coauthor of more than 110 papers in mathematical, technical journals, and conferences. He has been involved in more than 10 projects supported by the National Natural Science Foundation of China, the National Education Committee Foundation of China, and other important foundations.

His research interests include nonlinear control systems, control system design over network, teleoperation systems and intelligent control.

E-mail: cch@ysu.edu.cn (Corresponding author)

ORCID iD: 0000-0001-6311-2112

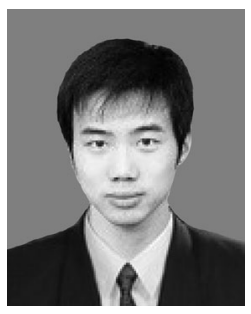

Shao-Chong Yu received his B. Sc. degree in electrical engineering from Yanshan University, China in 2011. He is currently a master student in electrical engineering.

His research interest is networked control system.

E-mail: yscysu@163.com

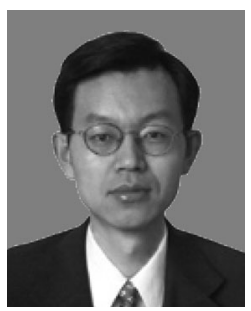

Xin-Ping Guan received the B. Sc. degree in mathematics from Harbin Normal University, China in 1986, received the M. Sc. degree in applied mathematics, and received the $\mathrm{Ph}$. D. degree in electrical engineering, both from Harbin Institute of Technology in 1991 and 1999, respectively. He is with the Department of Automation, Shanghai Jiao Tong University, China. He is the (co)author of more than 200 papers in mathematical, technical journals, and conferences. As an (co)-investigator, he has finished more than 20 projects supported by National Natural Science Foundation of China, the National Education Committee Foundation of China, and other important foundations. He is Cheung Kong Scholars Programme special appointment professor. He is serving as a reviewer of Mathematic Review of America, a member of the Council of Chinese Artificial Intelligence Committee, and the chairman of Automation Society of Hebei Province, China.

His research interests include networked control systems, robust control and intelligent control for complex systems and their applications.

E-mail: xpguan@ysu.edu.cn 